\section{Integrative Medicine \\ International}

\title{
Kidneys in Oriental and Occidental Medicine
}

\author{
Tetsuya Kondo \\ Kansai University of Health Sciences, Kumatori-cho, Japan
}

\section{Key Words}

Aging - Adrenal gland - Calcium metabolism - Alopecia - Inflammation - Diabetes mellitus . Osteoporosis - Alzheimer disease - Bio-mechanism - Brain - Emotion - High blood pressure $\cdot$ Traditional Chinese medicine

\section{Abstract}

Background: Oriental medicine is based on the 5-phase theory, in which the balance among the 5 viscera - the liver, the heart, the spleen, the lung and the kidney - is important. It is confusing that the viscera in Oriental and Occidental medicine have the same name. Out of the 5 viscera in Oriental medicine, it is the spleen that differs most from that in Occidental medicine. It mainly corresponds to the digestive organs and pancreas and has no relation to the spleen in Occidental medicine, a kind of lymph tissue. On the other hand, the concept of each of the other Oriental viscera includes the corresponding viscus in Occidental medicine. Key Messages: The 11 aspects of the kidney in Oriental medicine correspond to three kinds of endocrine and metabolic systems, all of which are associated with aging.

According to traditional Chinese theory, the kidney is a comprehensive system which regulates not only water-electrolysis metabolism but also glucose metabolism, food intake, emotion, especially fear, motivation, cognitive function, genital function, bone, hair and heat production (fig. 1).

Out of these functions, glucose metabolism, water-electrolysis metabolism and genital function are basic adrenocortical functions. According to traditional Chinese theory, diseases result from the imbalance among the 5 elements wood, fire, earth, metal and water. The kidney has the nature of water needed to extinguish the fire. Fire not only stands for inflammation but also for excitement of the central nervous system such as heat of anger. These functions are consistent with the role of glucocorticoids as the anti-inflammatory and antistress hormone. 


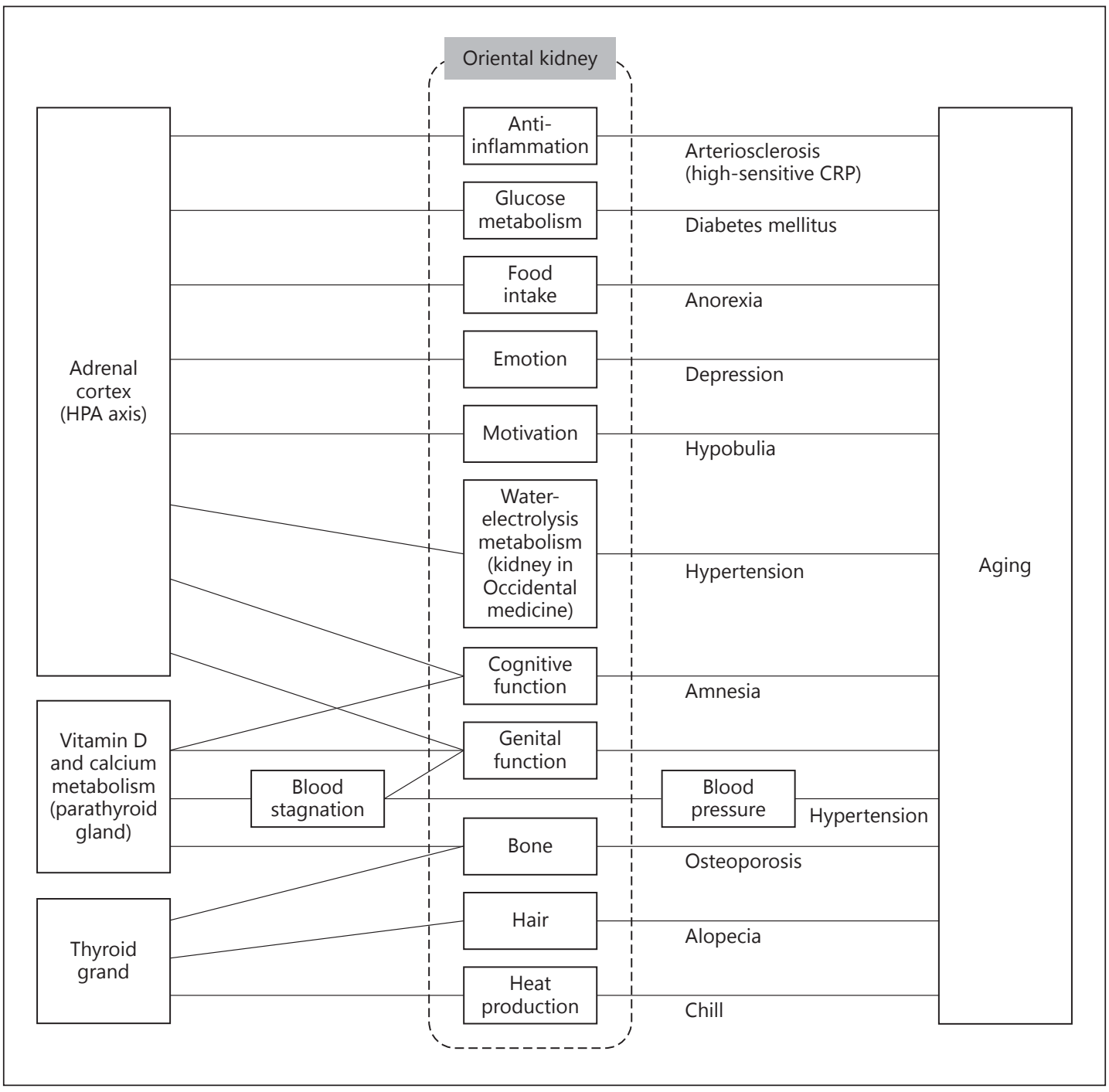

Fig. 1. Correspondence between the Occidental functions (left column) and the Oriental functions (median column) of the kidney. All the functions are associated with aging. CRP = C-reactive protein.

In our previous, large case-control study, a kind of kidney deficiency pattern according to traditional Chinese medicine was associated with eating disorder not otherwise specified [1]. It is possible that this disorder is a kind of chronic mild adrenocortical dysfunction, since anorexia can result from chronic adrenocortical dysfunction.

Recently, it has been reported that hippocampal atrophy and disturbed negative feedback in the hypothalamic-pituitary-adrenal (HPA) axis are associated with specific cognitive impairment in Alzheimer disease [2]. It was also reported that glucocorticoid sensitivity of the cognitive system is decreased in depression [3], which is a kind of emotional and cognitive disorder with decreased motivation. These indicate that the HPA axis is involved in emotion, motivation and cognitive function, and therefore, it is considered as the most important function of the kidney in Oriental medicine.

Out of the biological functions of the kidney in Oriental medicine, some are considered to be regulated by vitamin $\mathrm{D}$ and calcium metabolism. According to traditional Chinese theory, kidney dysfunction results in the dysfunction of what is generically named 'the medullas', 
which corresponds to bone marrow and brain medulla. This is consistent with the recent report that lower vitamin $\mathrm{D}$ concentrations are associated with poorer cognitive function and a higher risk of Alzheimer disease [4]. Hachimijiogan (Ba-Wei-Di-Huang-Wan) is a typical Kampo formula used to reinforce the function of the kidney in Oriental medicine and to prevent aging. This formula is effective not only in kidney patterns according to traditional Chinese medicine but also in renal dysfunction in Occidental medicine, whose serum creatinine is less than $3 \mathrm{mg} / \mathrm{dl}$ [5]. This indicates that the concepts of the kidney in Oriental and Occidental medicine are not very different from each other. It was reported that this formula improved the increase in urinary calcium excretion and the elevation of serum parathyroid hormone levels in aged rats [6]. These effects strengthen the bones throughout the body, which are administered by the kidney in Oriental medicine.

In addition to the medullas, the erythrocyte function is regulated by calcium. It was reported that calcium affects the membrane fluidity of the erythrocytes [7-9] through the intermediary of nitric monoxide or insulin [10]. Erythrocytes with decreased membrane fluidity would tend to grow stagnant inside the capillaries. This state is called blood stagnation. In fact, Terasawa [11] observed that the membrane fluidity of the erythrocytes was decreased in patients with blood stagnation, indicating that calcium metabolism may be associated with blood stasis. Assuming that the kidney is a calcium regulation system, this is consistent with the traditional Chinese theory that blood stagnation can result from a kidney deficiency pattern [12].

The calcium-dependent regulation of the erythrocyte membrane fluidity was disturbed in patients with essential hypertension [13]. For hypertension, especially in perimenopausal and postmenopausal women, a typical Kampo formula for blood stasis, Keishibukuryogan, was effective $[14,15]$. In addition, there is direct evidence for the involvement of the Oriental kidney in hypertension. Hachimijiogan mentioned above exhibited hypotensive effects [16].

According to traditional Chinese theory, 12 meridians run vertically between the head and the extremities under or on the body surface and regulate the function of the organs and the skeletal muscles on their way. For example, the kidney meridian runs from the feet to the tongue beside and most close to the ventral median line. On the way, this meridian runs through the thyroid gland and the parathyroid gland. Therefore, these two endocrine organs are administered by the kidney. Out of the elements administered by the kidney mentioned above, heat production is a fundamental function of the thyroid gland. The two elements of the kidney in Oriental medicine, bones and hair, are reported to be regulated by thyroid function. It was reported that subclinical hyperthyroidism is associated with fracture risk in women [17-19]. In addition, some alopecia derives from hyperthyroidism or Hashimoto's disease $[20,21]$.

According to traditional Chinese theory, the kidney is the organ most closely associated with aging. Aging is considered as kidney deficiency. In other words, aging is characterized by the immune, neural and endocrine dysfunctions mentioned above, such as diabetes mellitus, anorexia, hypobulia [22], cognitive dysfunction [23-25], hypertension [26], osteoporosis [25], alopecia [27] and chill [28], in addition to arteriosclerosis [29] resulting from slight inflammation [30]. In summary, the concept of the kidney in Oriental medicine is broader than that in Occidental medicine.

\section{Summary}

Of the 5 viscera, in Oriental medicine, the function of the kidney is the most complicated. However, its function is easily comprehensible in Occidental medicine. 
Kondo: Kidneys in Oriental and Occidental Medicine

\section{References}

1 Kondo T, Tokunaga S, Sugahara H, Yoshimasu K, Kanemitsu Y, Kubo C: Identification of visceral patterns in patients with stress-related disorders. Intern Med Int 2015;1:1-14.

2 Elgh E, Lindqvist Astot A, Fagerlund M, Eriksson S, Olsson T, Nasman B: Cognitive dysfunction, hippocampal atrophy and glucocorticoid feedback in Alzheimer's disease. Biol Psychiatry 2006;59:155-161.

3 Rohleder N, Wolf JM, Wolf OT: Glucocorticoid sensitivity of cognitive and inflammatory processes in depression and posttraumatic stress disorder. Neurosci Biobehav Rev 2010;35:104-114.

4 Balion C, Griffith LE, Strifler L, Henderson M, Patterson C, Heckman G, Llewellyn DJ, Raina P: Vitamin D, cognition, and dementia: a systematic review and meta-analysis. Neurology 2012;79:1397-1405.

5 Mitsuma T: Preventive effects of eastern medication (Kampo) on the progression of chronic renal failure. Int J Urol 1996;3:S95-S100.

6 Ikeda R, Mizoguchi K: Hachimijiogan (Ba-Wei-Di-Huang-Wan), a herbal medicine, improves unbalance of calcium metabolism in aged rats. J Ethnopharmacol 2009;124:176-181.

7 Muzulu SI, Bing RF, Norman RI: Human erythrocyte membrane fluidity and calcium pump activity in primary combined hyperlipidaemia. Clin Sci (Lond) 1995;88:307-310.

8 Watanabe H, Kobayashi A, Yamamoto T, Suzuki S, Hayashi H, Yamazaki N: Alterations of human erythrocyte membrane fluidity by oxygen-derived free radicals and calcium. Free Radic Biol Med 1990;8:507-514.

9 Sauerheber RD, Lewis UJ, Esgate JA, Gordon LM: Effect of calcium, insulin and growth hormone on membrane fluidity. A spin label study of rat adipocyte and human erythrocyte ghosts. Biochim Biophys Acta 1980;597: 292-304.

10 Brzeszczynska J, Gwozdzinski K: Nitric oxide induced oxidative changes in erythrocyte membrane components. Cell Biol Int 2008;32:114-120.

11 Terasawa K: Scientific approach to OKETSU (blood stasis) syndrome. Kampo Med 1998;48:409-436.

12 Gao W: Verses on One Hundred Symptoms (Hyakushofu). Tokyo, Souikai, 1976.

13 Tsuda K: Effects of the calcium channel blocker benidipine on membrane fluidity of erythrocytes in subjects with essential hypertension: an electron paramagnetic resonance study. Clin Exp Pharmacol Physiol 2007; 34(suppl 1):67-69.

14 Terauchi M, Akiyoshi M, Owa Y, Kato K, Obayashi S, Kubota T: Effects of the Kampo medication keishibukuryogan on blood pressure in perimenopausal and postmenopausal women. Int J Gynaecol Obstet 2011;114: 149-152.

15 Terauchi M, Hiramitsu S, Akiyoshi M, Owa Y, Kato K, Obayashi S, Matsushima E, Kubota T: Effects of three Kampo formulae: Tokishakuyakusan (TJ-23), Kamishoyosan (TJ-24), and Keishibukuryogan (TJ-25) on Japanese peri- and postmenopausal women with sleep disturbances. Arch Gynecol Obstet 2011;284:913-921.

16 Hirawa N, Uehara Y, Kawabata Y, Numabe A, Takada S, Nagoshi H, Gomi T, Ikeda T, Omata M: Hachimi-jio-gan extract protects the kidney from hypertensive injury in Dahl salt-sensitive rat. Am J Chin Med 1996;24:241254.

17 Wartofsky L: Subclinical hyperthyroidism and fracture risk in women. J Clin Endocrinol Metab 2014;99:26542656.

18 Polovina S, Micic D, Miljic D, Milic N, Micic D, Popovic V: The Fracture Risk Assessment Tool (FRAX score) in subclinical hyperthyroidism. Vojnosanit Pregl 2015;72:510-516.

19 Mukhopadhyay S, Satpathi T: Retrosternal goitre with subclinical hyperthyroidism presenting with trochanteric fracture. J Assoc Physicians India 2010;58:502-504.

20 Welti H: Skin manifestations associated with severe forms of Basedow's disease. Bull Schweiz Akad Med Wiss 1968;23:476-482.

21 Bianchi D, Masina M, Passarini B, Varotti C: Total alopecia and Basedow's disease (in Italian). Soc Ital Dermatol Sifilografia 1986;121:335-336.

22 Duzel E, Bunzeck N, Guitart-Masip M, Duzel S: Novelty-related motivation of anticipation and exploration by dopamine (NOMAD): implications for healthy aging. Neurosci Biobehav Rev 2010;34:660-669.

23 Angelucci L: Glucocorticoid hormone, aging brain and dementia. Funct Neurol 1997;12:167-173.

24 Glisky EL: Changes in Cognitive Function in Human Aging Brain Aging: Models, Methods, and Mechanisms. Boca Raton, Taylor \& Francis Group, 2007.

25 Cornelius C, Koverech G, Crupi R, Di Paola R, Koverech A, Lodato F, Scuto M, Salinaro AT, Cuzzocrea S, Calabrese EJ, Calabrese V: Osteoporosis and Alzheimer pathology: role of cellular stress response and hormetic redox signaling in aging and bone remodeling. Front Pharmacol 2014;5:120.

26 Fukutomi M, Kario K: Aging and hypertension. Expert RevCardiovasc Ther 2010;8:1531-1539.

27 Goodier M, Hordinsky M: Normal and aging hair biology and structure 'aging and hair'. Curr Probl Dermatol 2015;47:1-9.

28 Vogelaere P, Pereira C: Thermoregulation and aging. Rev Port Cardiol 2005;24:747-761.

29 Kunz J: Initial lesions of vascular aging disease (arteriosclerosis). Gerontology 2000;46:295-299.

30 Zhong J, Wang Y, Wang X, Li F, Hou Y, Luo H, Chen H: Significance of CAVI, hs-CRP and homocysteine in subclinical arteriosclerosis among a healthy population in China. Clin Invest Med 2013;36:E81-E86. 